\title{
Quantum statistics of a kicked particle in an infinite potential well
}

\author{
D. Kilbane ${ }^{\text {a,b,c }}$, A. Cummings ${ }^{\text {a }}$, G. O’Sullivan ${ }^{\text {a }}$, D.M. Heffernan ${ }^{\text {b,c,** }}$ \\ ${ }^{a}$ School of Physics, University College Dublin, Ireland \\ ${ }^{\mathrm{b}}$ Department of Mathematical Physics, National University of Ireland, Maynooth, Ireland \\ ${ }^{\mathrm{c}}$ School of Theoretical Physics, Dublin Institute for Advanced Studies, Dublin 4, Ireland
}

Accepted 11 January 2006

\begin{abstract}
It is known that no one statistical test by itself can give conclusive evidence for the presence or absence of quantum chaos within a given system. For this reason a range of detailed tests, namely the nearest neighbour spacing distribution, covariance of adjacent spacings, spectral rigidity, correlation-hole method and inverse participation ratio have been applied to the quasienergies and quasieigenstates of a periodically kicked particle in a 1-D infinite potential well. The results are compared with the predictions of random matrix theory for various kick strengths in order to search for signatures of quantum chaos within this system.
\end{abstract}

(C) 2006 Elsevier Ltd. All rights reserved.

\section{Introduction}

Investigation of non-analytic systems began with the kicked harmonic oscillator, which describes a charged particle moving in a magnetic field [1-9, and references therein]. However it is difficult to study quasienergies, Floquet states and the long time diffusion of this model [10-12], as its phase space is unbounded in position and momentum and cannot be reduced to a cylinder, as in the case of the kicked rotor [13]. For this reason $\mathrm{Hu}$ et al. [13] introduced the model of a kicked particle in an infinite potential well, which is bound in position. The quasieigenstates of this model showed quite different behaviour from that of the kicked rotator, in that they were found to be power-law localized, which was traced back to the structure of the evolution matrix [13]. The level statistics of the quasienergies displayed a smooth transition from a Poisson to a Wigner distribution for a fixed dimension of the floquet matrix [13].

In [14], a discontinuous generalization of the standard map, which arises naturally as the dynamics of a periodically kicked particle in an infinite potential well, was examined. The quantum dynamics of this system were also investigated [15] with particular emphasis on the localization properties of the quasieigenstates. The eigenstates were found to be more localized in the regular or mixed regimes of the classical system and delocalized in the chaotic regimes which are characterized by a log-normal distribution of the participation ratios. In the regular case the nearest neighbour spacing distribution of the quasienergies showed good agreement with the Poisson distribution [15]. In highly chaotic

\footnotetext{
* Corresponding author. Tel.: +353 1 7083775; fax: +3531 7083967.

E-mail address:dmh@thphys.may.ie (D.M. Heffernan).
} 
regimes the spacing did not show the RMT predicted Wigner distribution as was claimed in [13]. The authors concluded that while the spacing is not sensitive to classical chaos, the distribution of participation ratios of the eigenstates was found to be a good measure to distinguish chaotic quantum systems from regular ones [15].

In [16], Borgonovi introduced the discontinuous perturbed twist map which is an approximation of the stadium map [17]. The classical and quantum properties of this map were investigated and different localization regimes were found. Jie et al. [18] generalized these results where the power-law localization of the quasieigenstates in momentum space is found to be a universal feature of non-analytic systems, as opposed to the exponential localization of the quasieigenstates of the kicked rotor [19]. With increasing perturbation strength a transition from perturbative localization to pseudo-integrable system, to dynamical localization and to complete extension of the quasieigenstates was observed [18].

In this work a more extensive numerical investigation of the quasieigenvalues and quasieigenstates is presented than in previous studies. The evolution matrix of the system is derived in Section 2. In Section 3 the quasienergy level statistics are investigated with particular emphasis on the nearest neighbour spacing distribution, covariance of adjacent spacings, spectral rigidity and the correlation-hole method. These results are compared with the predictions of random matrix theory. The quasieigenstate statistics are discussed in Section 4 with particular emphasis on the structure of the quasieigenstates and the inverse participation ratio.

\section{The evolution matrix}

The Hamiltonian of the kicked particle in a 1-dimensional infinite potential well [13] is defined as

$$
\widehat{H}=\frac{\widehat{P}^{2}}{2 m}+V_{0}(q)+k \cos (q+\alpha) \sum_{n=-\infty}^{\infty} \delta(t-n T)
$$

where

$$
V_{0}(q)= \begin{cases}0, & 0<q<\pi \\ \infty, & \text { otherwise }\end{cases}
$$

$\alpha=1$ is a phase shift introduced to break the parity symmetry of the well, $T=1$ is the period of the kick and $k$ is the kick strength. The evolution operator of the system is given by [13]

$$
\widehat{U}=\exp \left(\frac{-\mathrm{i} \widehat{P}^{2} T}{4 \hbar}\right) \exp \left(\frac{-\mathrm{i} k \cos (q+\alpha)}{\hbar}\right) \exp \left(\frac{-\mathrm{i} \widehat{P}^{2} T}{4 \hbar}\right)
$$

Since this Floquet operator, $\widehat{U}$, is unitary, it satisfies the eigenvalue equation,

$$
\widehat{U}\left|\Psi_{\mathrm{f}}\right\rangle=\mathrm{e}^{-\mathrm{i} \omega_{n}}\left|\Psi_{\mathrm{f}}\right\rangle
$$

where $\omega_{n}$ is the quasienergy and $\Psi_{\mathrm{f}}$ the Floquet state (quasieigenstate). The above eigensystem may be solved by diagonalizing a matrix representation of $\widehat{U}$. The eigenstates of the unperturbed Hamiltonian $H_{0}$ were taken as the basis states for the $U$ matrix [13],

$$
H_{0}\left|\psi_{n}\right\rangle=E_{n}\left|\psi_{n}\right\rangle
$$

where $E_{n}$ is the $n$th energy eigenvalue. $\left|\psi_{n}\right\rangle$ are the eigenfunctions of the Hamiltonian $H_{0}$ given by [20]:

$$
\left\langle q \mid \psi_{n}\right\rangle=\sqrt{\frac{1}{a}} \sin \left(\frac{n \pi q}{2 a}\right), \quad E_{n}=\frac{\hbar^{2} n^{2} \pi^{2}}{8 m a^{2}}
$$

where $n=1,2,3 \ldots, N$. The particle is restricted between 0 and $\pi\left(2 a=\pi\right.$ is the width of the well). Since $E_{n}=P^{2} / 2 m$, and setting mass $m=1$ gives $P^{2}=\hbar^{2} n^{2}$. Substituting this into Eq. (2) yields

$$
\widehat{U}(T)=\exp \left(\frac{-\mathrm{i} \hbar\left(n^{2}+m^{2}\right) T}{4}\right) \exp \left(\frac{-\mathrm{i} k \cos (q+\alpha)}{\hbar}\right)
$$


The evolution matrix $U$ is then calculated as

$$
\begin{aligned}
U_{n m} & =\left\langle\psi_{n}|U| \psi_{m}\right\rangle=\frac{2}{\pi} \mathrm{e}^{\frac{-i \hbar h\left(n^{2}+m^{2}\right) T}{4}} \int_{0}^{\pi}\left[\left(\sin (n q) \sin (m q) \mathrm{e}^{\left.\frac{-i k \cos (q+\alpha)}{h}\right)}\right] \mathrm{d} q\right. \\
& =C \int_{0}^{\pi} \mathrm{e}^{\frac{-i k \cos (q+\alpha)}{h}}(\cos ((n-m) q)-\cos ((n+m) q)) \mathrm{d} q
\end{aligned}
$$

where the constant $C=\frac{1}{\pi} \mathrm{e}^{\frac{-\mathrm{i} h\left(n^{2}+m^{2}\right) T}{4}}$. Letting $z=-k / \hbar$, the integral reduces to

$$
\int_{0}^{\pi} \cos (\beta q) \mathrm{e}^{\mathrm{i} z \cos (q+\alpha)} \mathrm{d} q, \quad \text { where } \beta=n+m \quad \text { or } \quad \beta=n-m
$$

Employing the Bessel function $J_{n}(z)$ through the identity

$$
\mathrm{e}^{\mathrm{i} z \cos (q+\alpha)}=\sum_{l=0}^{\infty} \varepsilon_{l} i^{l} J_{l}(z) \cos (l(q+\alpha))
$$

where $\varepsilon_{0}=1$; and $\varepsilon_{l}=2$ for $l \geqslant 1$ [21], the integral is evaluated by expanding as a series

$$
\int_{0}^{\pi}\left[\cos (\beta q) \sum_{l=0}^{\infty} \varepsilon_{l} l^{l} J_{l}(z) \cos (l(q+\alpha))\right] \mathrm{d} q=\sum_{l=0}^{\infty} \varepsilon_{l} l^{l} J_{l}(z) \int_{0}^{\pi} \cos (\beta q) \cos (l(q+\alpha)) \mathrm{d} q
$$

The analytical result for the matrix elements of $U$ are thus as follows:

$$
\begin{aligned}
U_{n m}= & C \int_{0}^{\pi} \cos (\beta q) \mathrm{e}^{\mathrm{i} z \cos (q+\alpha)} \mathrm{d} q \\
= & C\left[\pi \mathrm{i}^{\beta} J_{\beta}(z) \cos (\beta \alpha)-4 \sum_{l=2,4}^{\infty} \mathrm{i}^{l} J_{l}(z) \sin (l \alpha)\left(\frac{l}{l^{2}-\beta^{2}}\right)\right] \text { for } \beta \text { odd } \\
& +C\left[\pi \mathrm{i}^{\beta} J_{\beta}(z) \cos (\beta \alpha)-4 \sum_{l=1,3}^{\infty} \mathrm{i}^{l} J_{l}(z) \sin (l \alpha)\left(\frac{l}{l^{2}-\beta^{2}}\right)\right] \text { for } \beta \text { even }
\end{aligned}
$$

\section{Quasienergy level statistics}

Diagonalization of the evolution matrix gives a set of quasienergies that can be compared with the predictions of RMT. Due to the overall symmetry properties of the evolution matrix, the results of the statistical tests are compared with the circular orthogonal ensemble (COE) predictions of RMT [22]. In the limit of large $N$ these statistics are the same as predicted for the Gaussian orthogonal ensembles (GOE) [22]. In our present study we have used, as in [13], a matrix dimension of $N=1024$.

\subsection{Rescaling of the quasienergy levels}

Before studying the various level statistics, the quasieigenvalues were rescaled by applying two transformations: (i) the phases of the eigenvalues were rescaled to $\operatorname{Re}\left(\omega_{n}\right) \frac{N}{2 \pi}$ and (ii) a linear transformation was applied using the fitting parameters given in Table 1. This results in an average level density (global) of 1 for the rescaled $\omega_{n}$.

Table 1

Various curve fitting parameters of the quasienergy statistics

\begin{tabular}{llrrr}
\hline \multicolumn{1}{l}{$k$} & & & \\
\cline { 2 - 5 } & 0.1 & 5 & 0.99 & 50 \\
\hline$m$ & 0.98 & -0.30 & 1.94 & 0.86 \\
$c$ & 8.40 & 1.10 & 1.40 & -2.91 \\
$1+\Omega$ & 1.07 & 0.10 & 0.40 & 1.75 \\
$\Omega$ & 0.07 & 0.032 & -0.06 & 0.75 \\
Covariance of adjacent spacings & 0.07 & & & -0.10 \\
\hline
\end{tabular}




\subsection{Nearest neighbour spacing distribution}

The nearest neighbour spacing (NNS) distribution, $P(s)$, is defined as the probability density for two neighbouring levels $x_{n}$ and $x_{n+1}$ to be separated by a distance $s$ on the rescaled scale. It is a short-range spectral fluctuation measure, i.e. only correlations between eigenvalues which are separated by a few other eigenvalues are involved. Using the rescaled quasienergies the NNS distributions were calculated and are shown in Fig. 1. The Wigner and Poisson distributions represent the extreme cases of classical behaviour i.e. regular (Poisson) and chaotic (Wigner). The smooth transition observed in [13] from Poisson at small $k$ to Wigner at large $k$ is clearly seen. Such a transition was only observed in [15] for relaxed convergence criterion.

In reality a system has usually a mixture of both regular and chaotic characteristics and the Brody distribution [23-26] is used to investigate this:

$$
P(x, \Omega)=\alpha(\Omega) \cdot(\Omega+1) \cdot x^{\Omega} \cdot \mathrm{e}^{-\alpha(\Omega) \cdot x^{\Omega+1}}, \quad \alpha(\Omega)=\Gamma\left(\frac{\Omega+2}{\Omega+1}\right)^{\Omega+1}
$$

In order to obtain the Brody parameter $\Omega$, a graph of $\ln \left(\ln \left[(1-\Pi(s))^{-1}\right]\right)$ versus $\ln (s)$ is plotted and $\Omega$ is found from its slope of $(1+\Omega)[27,26]$ where $\Pi(s)$ is the cumulative spacing distribution,

$$
\Pi(s)=\int_{0}^{s} P(x) \mathrm{d} x \equiv 1-\mathrm{e}^{-\alpha(\Omega) \cdot s^{2+1}}
$$

Fig. 1 shows $\Pi(s)$ and $\ln \left(\ln \left[(1-\Pi(s))^{-1}\right]\right)$ versus $\ln (s)$. The resulting $(1+\Omega)$ and hence $\Omega$ are given in Table 1 . The value of $\Omega$ for small $k$ indicates behaviour very close to Poissonian $(\Omega=0)$. The value of $\Omega$ for large $k$ is more Wignerlike level repulsion $(\Omega=1)$. The value of $\Omega$ for intermediate $k$ is indicative of intermediate level statistics i.e. the Brody distribution. Note the depression of $P(s)$ for $k=0.1$ and 5 for $s \rightarrow 0$, observed also in [12].

\subsection{Covariance of adjacent spacings}

Another test for possible GOE behaviour is the covariance of adjacent spacings [28,22]:

$$
\operatorname{cov}\left(s_{n}, s_{n+1}\right)=\frac{\sum_{n=1}^{N}\left(s_{n}-\left\langle s_{n}\right\rangle\right)\left(s_{n+1}-\left\langle s_{n+1}\right\rangle\right)}{\left[\left(\sum_{n=1}^{N}\left(s_{n}-\left\langle s_{n}\right\rangle\right)^{2}\right)\left(\sum_{n=1}^{N}\left(s_{n+1}-\left\langle s_{n+1}\right\rangle\right)^{2}\right)\right]^{1 / 2}}
$$

This is sensitive to correlations between levels $E_{i}$ and $E_{i+2}(s$ is measured on the rescaled 'energy' scale) and is predicted by GOE theory to have the value -0.27 [29]. The Poisson value is 0 i.e. no correlations. Table 1 shows that as $k$ increases the value becomes closer to that predicted by RMT, but even for $k=50$ the value is still significantly different from the RMT prediction.

\subsection{Spectral rigidity}

The spectral rigidity $\Delta_{3}(L)$ is used to distinguish classically chaotic behaviour from regular behaviour in the semiclassical limit. $\Delta_{3}(L)[30]$ is defined for the interval $[a, a+L]$ in the sequence $\left\{x_{i}\right\}$ as

$$
\triangle_{3}(a, L)=\frac{1}{L} \min _{A, B} \int_{a}^{a+L}[n(x)-(A x+B)]^{2} \mathrm{~d} x
$$

where $n(x)$ is the cumulative number of 'states' of the sequence $\left\{x_{i}\right\}$ [31,32]. For a regular quantum system the energy levels are distributed randomly and without correlation. A random sequence of uncorrelated levels has a soft character (linear dependence on $L$ i.e. $\Delta_{3}(L)=L / 15$ ). In contrast the energy levels of a chaotic quantum system exhibit strong correlations owing to the repulsion between the random matrix eigenvalues. A random matrix spectrum has a rigid character (a logarithmic dependence on $L$, i.e. for $L \gg 1, \Delta_{3}(L)=\frac{1}{\pi^{2}}\left(\ln (2 \pi L)+\gamma+1-\frac{5}{4}-\frac{\pi^{2}}{8}\right)$ for GOE matrices, where $\gamma$ is Euler's constant) $[22,33,34]$. The difference between the two spectra is most pronounced in the $\Delta_{3}(L)$ statistic since this involves correlations between many levels i.e. they test for long-range spectral fluctuations.

The spectral rigidity is shown in Fig. 2 (for $L=0 \rightarrow 50$ ), where \langle\rangle indicates spectral averaging [31,32]. It is seen from these figures that for small $k(k=0.1$ and 5), the spectrum follows the Poisson prediction for a limited range of $L$ $(L \approx 10)$ and then the spectrum becomes less rigid than a Poisson spectrum. This less than Poisson rigidity is due to matrix truncation. As $k$ increases the spectrum undergoes a transition and at large $k=50$ follows the GOE prediction for $L \leqslant 15$. For $L>15$ and $k=50$, the spectrum follows that of an uncorrelated Wigner spectrum which was found 

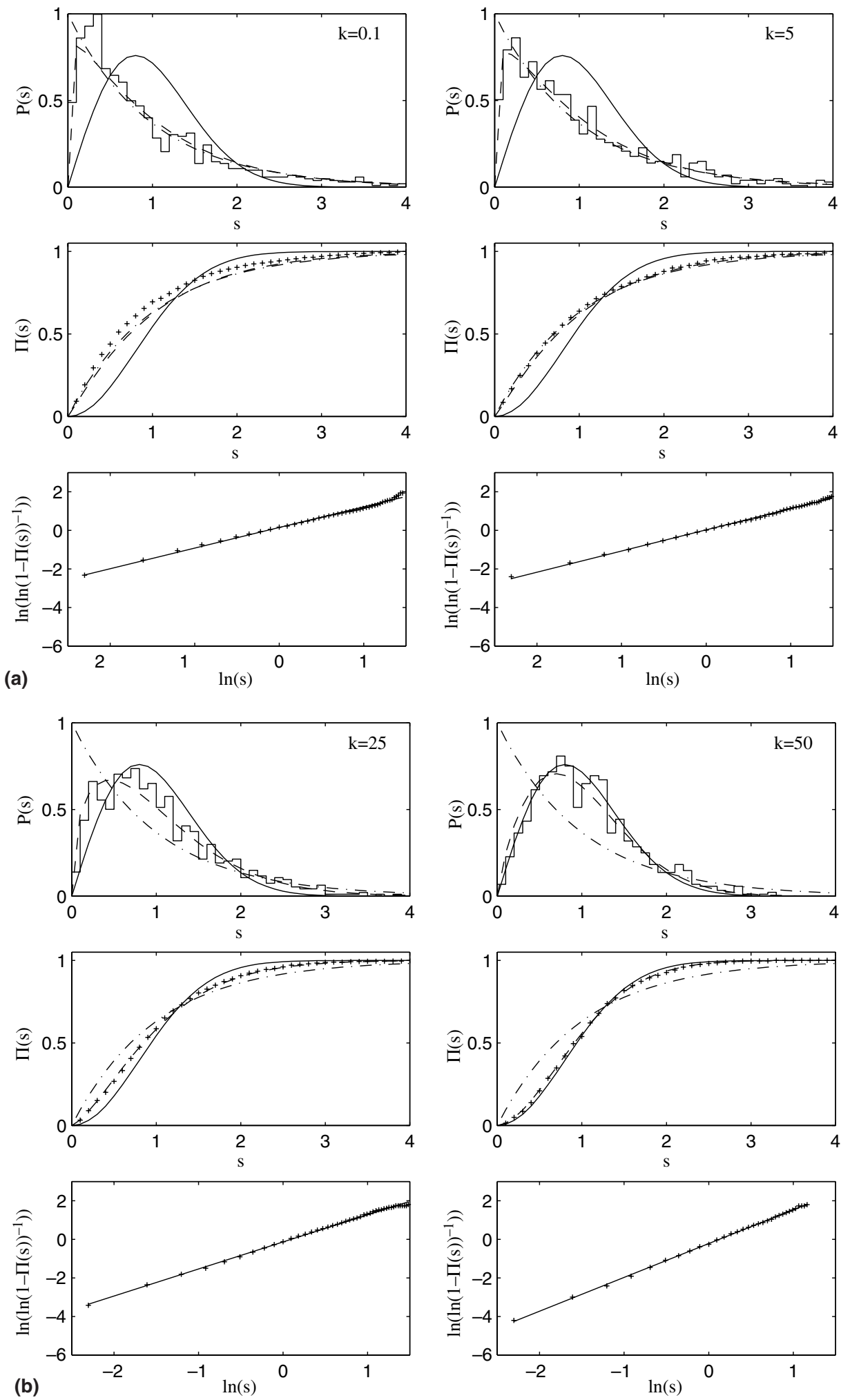

Fig. 1. $P(s), \Pi(s)$ and $\ln \left(\ln (1-\Pi(s))^{-1}\right)$, as kick strength is increased from $k=0.1$ to 50 . The Poissonian distribution (dash-dot), the Wigner distribution (solid) and the Brody distribution (dash) are also shown. 

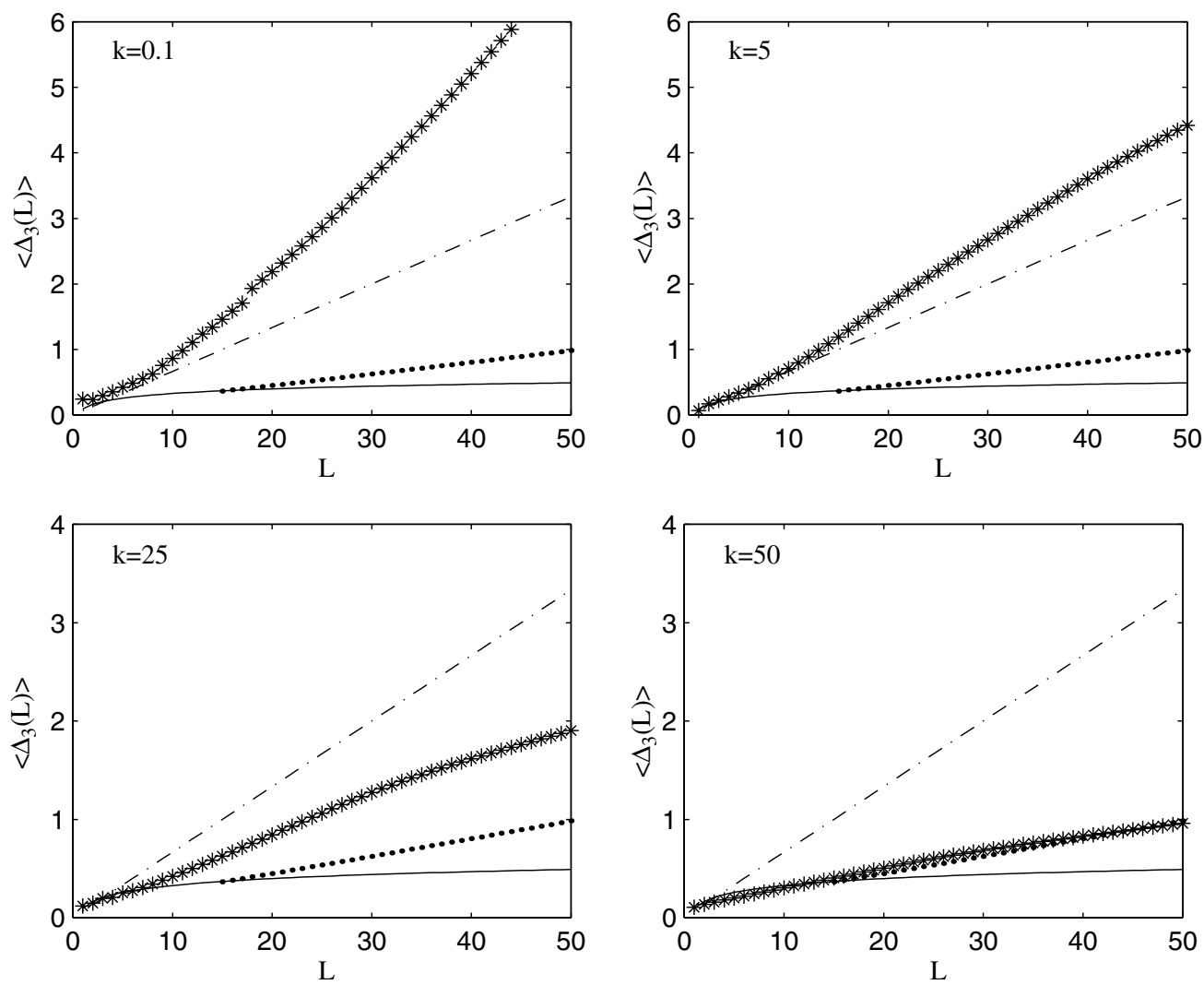

Fig. 2. Spectral rigidity $\Delta_{3}(L)$ and the GOE predictions, Wigner (solid), Poisson (dash-dot), uncorrelated Wigner (dot), as kick strength is increased from $k=0.1$ to 50 .

numerically to be equal to $\Delta_{3}(L)=L /[55-(210 / L)]$ for $L>15$ [35]. This intermediate behaviour between Poisson and GOE is suggestive, classically, of a mixed phase space of regular and chaotic behaviour [36].

In the limit of large $L(L \geqslant 50)$, the $\Delta_{3}(L)$ statistic cannot be explained and seems to be caused by large scale fluctuations of the cumulative level density. Experiments on quantum billiards for which ensembles of eigenvalues can be generated, have shown that when drawing only a single matrix from the ensemble, the $\Delta_{3}(L)$ statistic fluctuates considerably around the GOE prediction [37]. However taking the average over the ensemble reduces these fluctuations and the $\Delta_{3}(L)$ statistic follows closely that predicted by RMT. When dealing with a unique matrix $U$, spectral averaging, \langle\rangle , is performed to lessen the effects of these fluctuations. Another form of averaging, which may reduce fluctuations still further, is to average over a number of matrices with slightly different $k(\Delta k \ll k)$. This method was used in [19], for investigating the statistical properties of the quantum kicked rotor on a torus.

\subsection{Correlation-hole method}

Leviandier et al. [38] developed the correlation-hole method for the analysis of long-range correlations. The properly smoothed Fourier transform $C(t)$ of the spectral autocorrelation function maps the long-range correlations onto scales in Fourier space (time domain), i.e. $C(t)$ can be written as the Fourier transform of the autocorrelation function of a spectrum $I(x)$ on the rescaled scale $x[39]$ :

$$
C(t)=\int_{-\infty}^{+\infty} A(r) \mathrm{e}^{2 \pi \mathrm{i} r t} \mathrm{~d} r
$$

where $A(r)=\int_{-\infty}^{+\infty} I\left(R-\frac{r}{2}\right) I\left(R+\frac{r}{2}\right) \mathrm{d} R$ is the autocorrelation function of $I(x)$. In the case of a 'stick' spectrum of equal amplitudes $S(E)=\sum_{v} \delta\left(E-E_{v}\right)$ then [39]: 

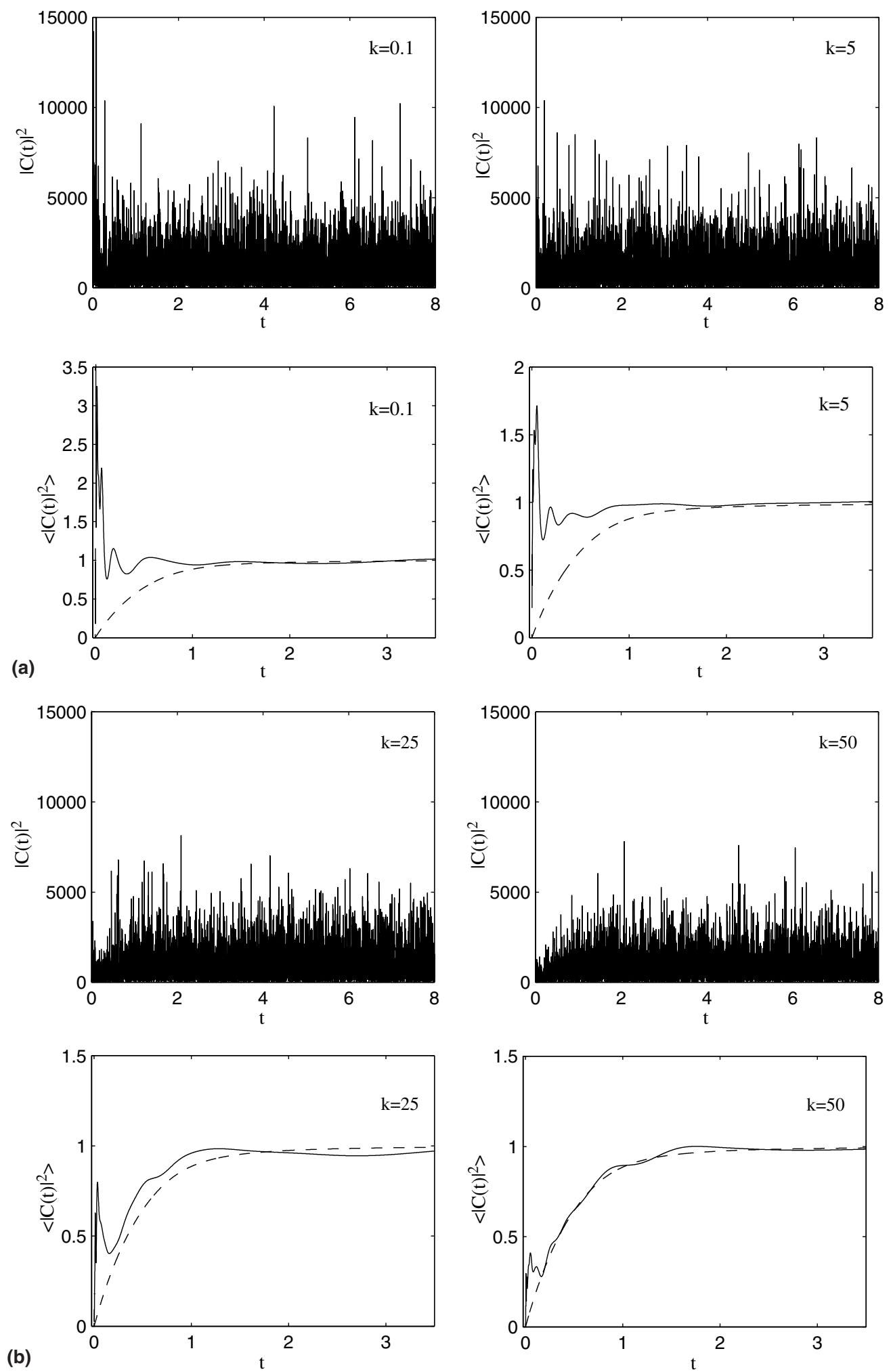

Fig. 3. Spectral form factor $|C(t)|^{2}$, averaged spectral form factor $\left\langle|C(t)|^{2}\right\rangle$ and the GOE predictions (dash), as kick strength is increased from $k=0.1$ to 50 . 


$$
|C(t)|^{2}=\left|\sum_{j, k} \mathrm{e}^{2 \pi i\left(E_{j}-E_{k}\right) t}\right|^{2}
$$

The ensemble average can be simulated by applying a smoothing procedure to the experimental decay function $|C(t)|^{2}$ [39]. The most appropriate procedure, known as 'full Gaussian smoothing', is a convolution of $|C(t)|^{2}$ with a Gaussian [39]. Hence, one has to compare the smoothed result to the function

$$
\left\langle|C(t)|^{2}\right\rangle=\int_{-\infty}^{+\infty}\left|C\left(t^{\prime}\right)\right|^{2} \frac{1}{\sqrt{2 \pi \sigma_{t}^{2}}} \exp \left(\frac{-\left(t-t^{\prime}\right)^{2}}{2 \sigma_{t}^{2}}\right) \mathrm{d} t^{\prime}
$$

where the variance was chosen to depend on the time as $\sigma_{t}=t / 5$. The theoretical $\left\langle|C(t)|^{2}\right\rangle$ is given by [39]

$$
\left\langle|C(t)|^{2}\right\rangle=N^{2}\left(\frac{\sin (\pi N t)}{\pi N t}\right)^{2}+N-N b_{2}(t)
$$

where $b_{2}(t)$ is the two-level form factor [22,34] and $N$ is the number of levels in the spectrum. Chaotic dynamics causes a suppression of the Fourier transform Eq. (17) near the origin, a so-called 'correlation hole'. For cases intermediate between fully chaotic and regular systems, the correlation hole is less deep and/or abrupt. In particular a Poisson spectrum should show no correlation hole $[38,40,26]$.

The $\mid$ Fourier transform $\left.\right|^{2},|C(t)|^{2}$ and $\left\langle|C(t)|^{2}\right\rangle$ of the rescaled quasienergies is shown in Fig. 3. The theoretical $\left\langle|C(t)|^{2}\right\rangle$ shown in the figures was also Gaussian smoothed. No correlation hole is present for small $k$ indicating regular dynamics. As $k$ increases a correlation hole appears which increases in size as $k$ becomes larger. This hole can indicate the presence of quantum chaos. However it should be noted that false correlation holes can arise even for regular systems [41], for example, in uncorrelated Wigner spectra. The appearance of the correlation hole as $k$ increases is
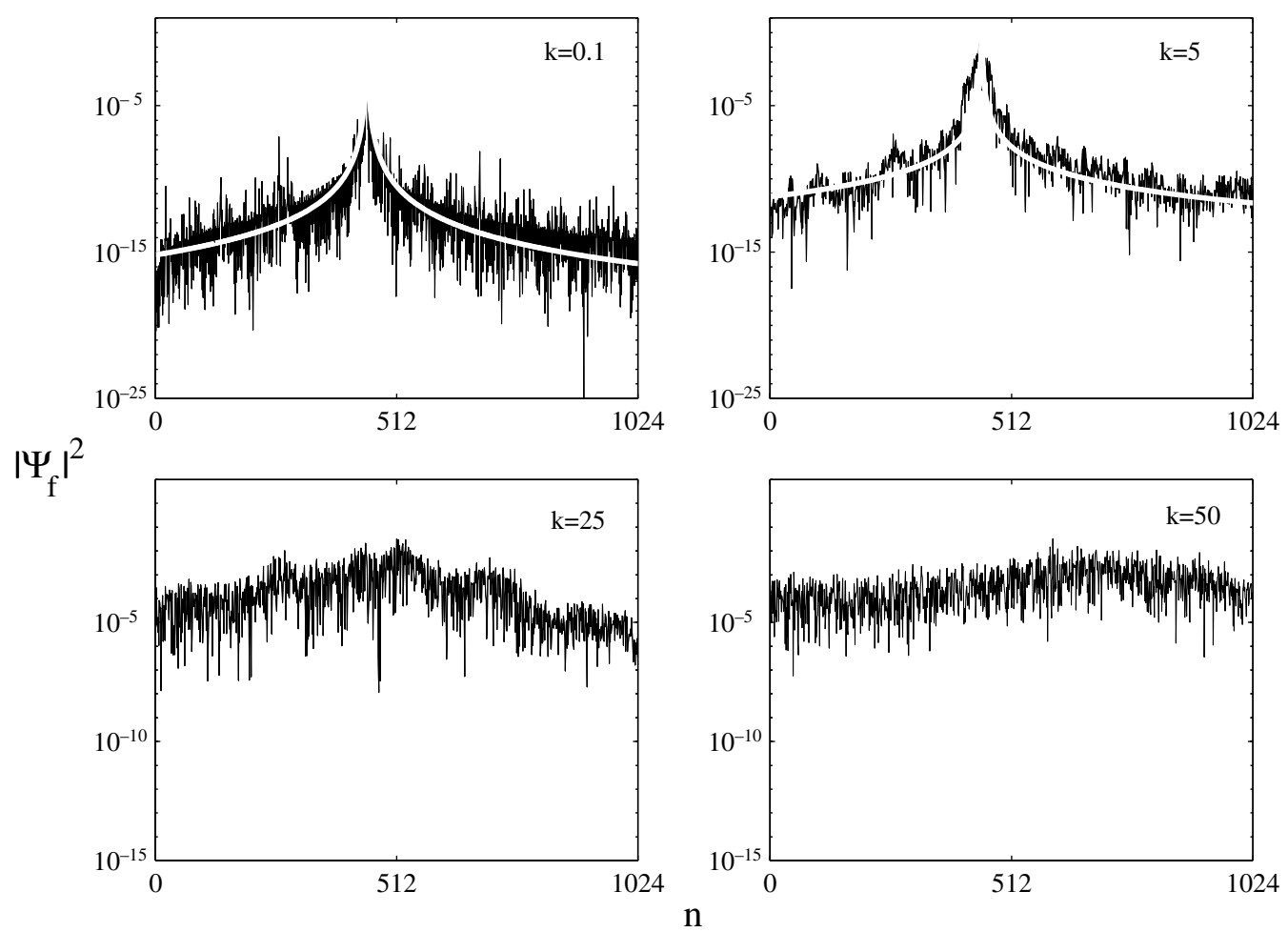

Fig. 4. Typical quasieigenstates in the different regimes: localized $(k=0.1$ and 5) fitted using Eq. (20), intermediate $(k=25)$ and extended $(k=50)(\log$-scale). 

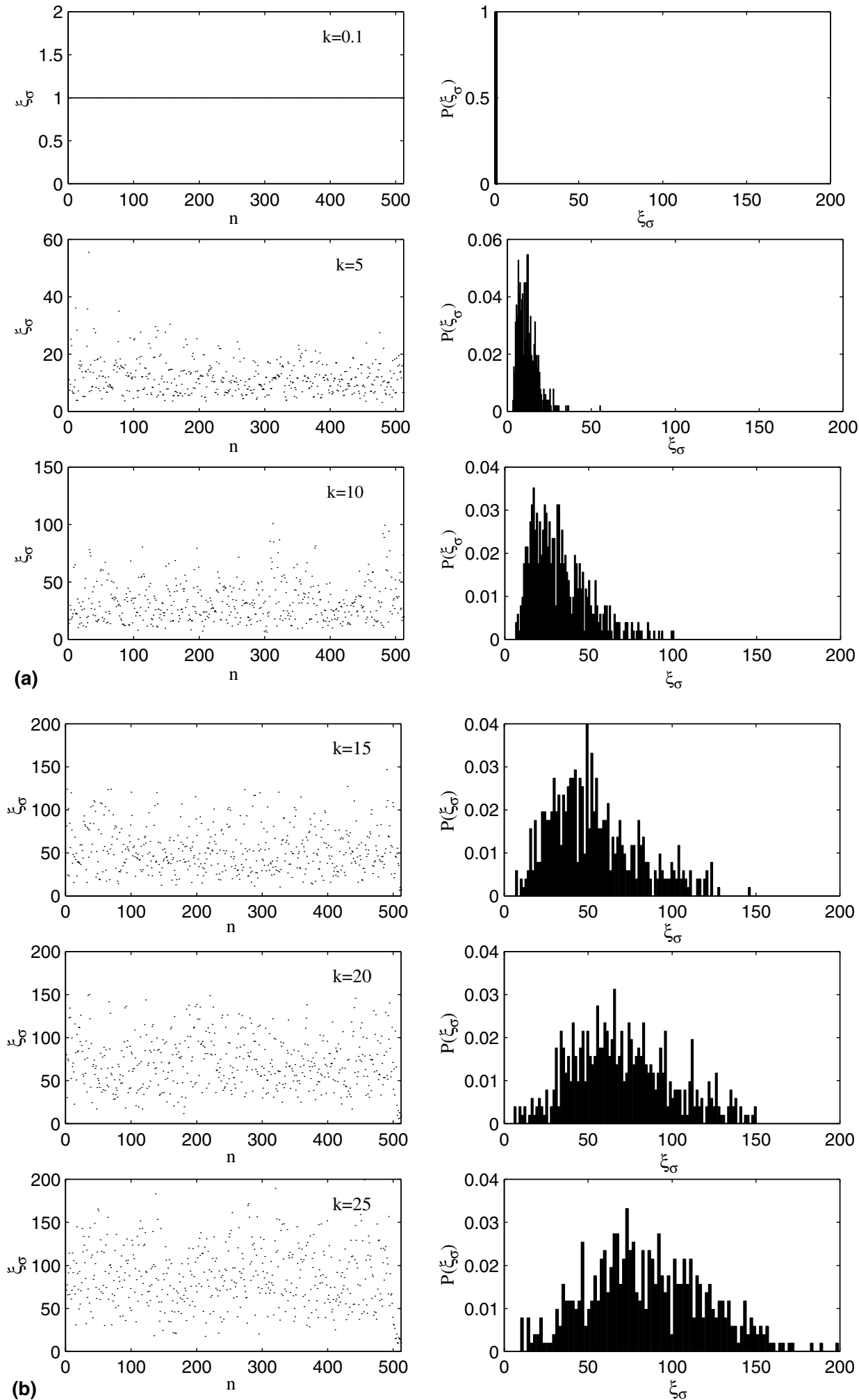

Fig. 5. Inverse participation ratio $\xi_{\sigma}$ as a function of $n$ and the probability distribution of the inverse participation ratios for various $k$. 
consistent with the behaviour found for $\left\langle\Delta_{3}(L)\right\rangle$ since small $t$ corresponds to large correlation lengths $L$ in $\left\langle\Delta_{3}(L)\right\rangle$. The presence of highly oscillatory small time behaviour in $\left\langle|C(t)|^{2}\right\rangle$ may indicate the effect of non-universal behaviour in the $\left\langle\Delta_{3}(L)\right\rangle$ statistic. This however may have its origin in the finite resolution and/or the rescaling procedure associated with missing energy levels [41].

\section{Quasieigenstate statistics}

The quasieigenstates show behaviour quite different from that of the kicked rotor. In the kicked rotor, the quasieigenstates are exponentially localized in momentum space $[42,43,18]$. In this model the quasieigenstates are power-law localized [13], for small $k$ values, as shown in Fig. 4. It is clearly seen that the localized states gradually transit to extended ones as $k$ increases.

\subsection{Structure of the quasieigenstates}

The power-law type localization is universal for non-analytic systems just like the exponential localization in analytic models [18]. In the kicked rotor the eigenstates are exponentially localized in the momentum space. For non-analytic systems, for a small perturbation $(k=0.1)$, i.e. in the perturbative regime, the envelope of a Floquet state, $\left|\Psi_{\mathrm{f}}\right\rangle$, in momentum representation can be fitted by the Lorentzian-like function

$$
\left|\Psi_{\mathrm{f}}\right|^{2} \simeq \frac{1}{1+4\left|n-n_{\mathrm{c}}\right|^{\gamma}}
$$

where $n_{\mathrm{c}}$ is the centre of the peak and $\gamma$ is a fitting parameter [18]. The power-law localization is clearly demonstrated in Fig. 4 for $k=0.1$, where the power exponent $\gamma \approx 5$.5. For $k=5$, the tails of the Floquet states are found to decay, on average, as a power-law with power exponent $\gamma \approx 3$.6. A similar study was undertaken in [15]. The results indicate that for large kick strengths, the 'well converged' states exhibit strong fluctuations in the basis but appear to have exponential tails.

\subsection{Inverse participation ratio $\xi_{\sigma}$}

To give a quantitative description of the localization phenomenon in non-analytic systems, the quasieigenstates are characterized by their inverse participation ratio as defined in [44],

$$
\xi_{\sigma}=\frac{\sum_{n}\left|\Psi_{\mathrm{f}}\right|^{2}}{\sum_{n}\left|\Psi_{\mathrm{f}}\right|^{4}}
$$

The behaviour of the inverse participation ratio for different values of $k$ corresponding to the localized and delocalized regimes are shown in Fig. 5.

For small $k$ it is seen that $\xi_{\sigma}=1$ for all quasieigenstates $n$. This is expected since all quasieigenstates are localized. For large $k(k=25)$, there is a spread in the $\xi_{\sigma}$ and a shift of the average $\xi_{\sigma}$ to $\sim 80$, indicating that the states are becoming more extended. The maximum value for $\xi_{\sigma}$ for a uniform probability distribution is given by $N$, where $N=512$ is the matrix dimension. Therefore as states get more extended, $\xi_{\sigma}$ approaches $N$. In this work, in contrast with [44], all quasieigenstates, including asymmetric states, were included in the calculation of $\xi_{\sigma}$, which may lead to a lower value of maximum $\xi_{\sigma}$. Also shown in Fig. 5 is the statistical distribution of the inverse participation ratios $P\left(\xi_{\sigma}\right)$ for localized states as well as extended states. In the localized regime the distribution is very narrow and the sharp peaks correspond to an accumulation of eigenstates with approximately the same value of $\xi_{\sigma}$. In contrast the mixed regime is characterized by a much broader distribution in $\xi_{\sigma}$, with a shift in the average value of $\xi_{\sigma}$.

\section{Conclusion}

The aim of the present paper was to find signatures of chaos at the quantum level for the model of a periodically kicked particle in an infinite potential well. Expanding the evolution matrix in a suitable basis produces a set of quasienergy levels and Floquet states that could statistically be compared with the predictions of random matrix theory. Owing to the symmetry properties of the evolution matrix these statistics are compared with the circular orthogonal ensembles, which approach the Gaussian orthogonal ensembles as $N \rightarrow \infty$. 
In [13], a transition was observed in the level spacing distribution from Poisson to Wigner as the perturbation was increased. However a large number of statistical tests are required in order to draw any decisive conclusions when searching for chaos at the quantum level. Here, a thorough investigation of the quasienergy level statistics has been performed. As $k$ increased, a spectral transition from Poisson (regular) to Wigner (chaotic) was observed in the nearest neighbour spacing distribution; the $\Delta_{3}$ statistic changed from that of a Poisson spectrum to an uncorrelated Wigner spectrum; and a correlation hole appeared. These results are characteristic of a classically mixed phase space containing chaotic and regular regions. This is in agreement with the classical phase space discussed in [45]. Lack of agreement between some of the quasienergy level tests and the predictions of random matrix theory can be attributed to the system never being fully ergodic and also to the finite size effects of $U$. The eigenstates were characterized by the inverse participation ratio. The Floquet states were found to be power-law localized for small $k$ and extended for large $k$.

\section{Acknowledgements}

This work was supported by the Irish Science and Technology Agency Enterprise Ireland under research grant SC/99/206.

\section{References}

[1] Zaslavsky GM, Zakharov MYu, Sagdeev RZ, Usikov DA, Chernikov AA. Zh Eksp Teor Fiz 1986;91:500.

[2] Zaslavsky GM, Zakharov MYu, Sagdeev RZ, Usikov DA, Chernikov AA. Sov Phys JETP 1986;64:294.

[3] Chernikov AA, Sagdeev RZ, Usikov DA, Zakharov MYu, Zaslavsky GM. Nature 1987;326:559.

[4] Chernikov AA, Sagdeev RZ, Zaslavsky GM. Physica 1988;33D:65.

[5] Zaslavsky GM, Sagdeev RZ, Usikov DA, Chernikov AA. Weak chaos and quasi-regular patterns. Cambridge University Press; 1991.

[6] Berman GP, Rubaev VYu, Zaslavsky GM. Nonlinearity 1991;4:543.

[7] Lichtenberg AJ, Wood BP. Phys Rev A 1989;39:2153.

[8] Dana I, Amit M. Phys Rev E 1995;51:R2731.

[9] Daly HV, Heffernan DM. Acta Phys Pol A 1996;89:571.

[10] Shepelyansky DL, Sire C. Europhys Lett 1992;20:95.

[11] Borgonovi F, Rebuzzini D. Phys Rev E 1995;52:2302.

[12] Hu B, Li B, Liu J, Zhou J-L. Phys Rev E 1998;58:1743.

[13] Hu B, Li B, Liu J, Gu Y. Phys Rev Lett 1999;82:4224.

[14] Sankaranarayanan R, Lakshminarayan A, Sheorey VB. Phys Lett A 2001;279:313.

[15] Sankaranarayanan R, Lakshminarayan A, Sheorey VB. Phys Rev E 2001;64:046210.

[16] Borgonovi F. Phys Rev Lett 1998;80:4653.

[17] Borgonovi F, Conti P, Rebuzzi D, Hu B, Li B. Physica D 1999;131:317.

[18] Jie L, TaiWang D, ShiGang C. Commun Theor Phys (Beijing, China) 2000;33:15.

[19] Izrailev FM. Phys Rep 1990;196:299.

[20] Richtmyer FK, Kennard EH, Cooper JN. Introduction to modern physics 6e. New Delhi: Tata McGraw-Hill; 1991.

[21] Dwight HB. Tables of integrals and other mathematical data. New York: The Macmillan Company; 1968.

[22] Mehta ML. Random matrices. 2nd ed. New York: Academic; 1991.

[23] Connerade JP. J Phys B 1997;30:L31.

[24] Brody TA. Lett Nuov Cim 1973;7:1482.

[25] Connerade JP, Grant IP, Marketos P, Oberdisse J. J Phys B 1995;28:2539.

[26] Cummings A, O’Sullivan G, Heffernan DM. J Phys B 2001;34:3447.

[27] Badrinarayanan R, José JV. In: Casati G, Chirikov BV, editors. Quantum chaos between order and disorder. Cambridge University Press; 1995.

[28] Porter CE. Statistical theories of spectra: fluctuations. New York: Academic Press; 1965.

[29] Camarda HS, Georgopulos PD. Phys Rev Lett 1983;50:492.

[30] Dyson FJ, Mehta ML. J Math Phys 1963;4:701.

[31] Bohigas O, Giannoni MJ. Ann Phys (NY) 1975;89:393.

[32] Ormond WE, Broglia RA. Phys Rev C 1992;46:1710.

[33] Pandey A. Ann Phys 1979;119:170.

[34] Guhr T, Müller-Groeling A, Weidenmüller HA. Phys Rev 1998;299:190.

[35] Camarda HS, Liou HI, Rahn F, Hacken G, Slagowitz M, Havens WW, et al. In: Garg JB, editor. Statistical properties of nuclei. New York: Plenum Press; 1972.

[36] Percival IC. Adv Chem Phys 1977;36:1. 
[37] Pedersen HJ. Random quantum billiards. MSc in Physics, Niels Bohr Institute, Copenhagen, 1997.

[38] Leviandier L, Lombardi M, Jost R, Pique JP. Phys Rev Lett 1986;56:2449.

[39] Delon A, Jost R, Lombardi M. J Chem Phys 1991;95:5701.

[40] Lombardi M, Bohigas O, Seligman TH. Phys Lett B 1994;324:263.

[41] Lombardi M, Labastie P, Bordas MC. J Chem Phys 1979;89:3479.

[42] Casati G, Chirikov BV, Ford J, Izrailev FM. Lecture Notes Phys 1979;93:334.

[43] Chirikov BV. Phys Rep 1979;52:263.

[44] Borgonovi F, Shepelyansky D. Europhys Lett 1995;29:117.

[45] Kilbane D, Cummings A, O'Sullivan G, Heffernan DM. The classical-quantum correspondence of a kicked particle in an infinite potential well. Chaos, Solitons \& Fractals, in press, doi:10.1016/j.chaos.2006.01.011. 